\title{
Reduced Basis Method for Quadratically Nonlinear Transport Equations
}

\author{
Nadine Jung $^{1} \quad$ B. Haasdonk ${ }^{2} \quad$ D. Kroener ${ }^{3}$
}

With the Reduced Basis Method (RBM) we can solve a given parametrized PDE for many parameters which are not known in advance faster then by Finite Element Method (FEM). Initially we choose in a suitable way $N$ parameters $\mu_{1}, \ldots, \mu_{N}$ within a certain interval and compute the FE solution $u_{H}\left(\mu_{i}\right)$ for $1 \leq i \leq N$ on an extremely fine mesh. The number $N$ is small compared to the dimension of the FE space. We construct a new space $X_{N}$, spanned by these $N \mathrm{FE}$ approximations. Then, in order to compute approximations for many different parameters $\mu$, different from $\mu_{1}, \ldots, \mu_{N}$, we compute an RB approximation $u_{N}(\mu)$ in the lower dimensional space $X_{N}$. Because of the lower dimensional space $X_{N}$ the computations of the RB approximation $u_{N}(\mu)$ is faster than the computation of the FE approximation $u_{H}(\mu)$. In [2] the RBM is applied to the stationary, viscous Burgers equation. [2] contains an a posteriori error estimator between $u_{N}(\mu)$ and $u_{H}(\mu)$ as well as an existence proof. The results of [2] are extended in [1] to a wider class of parameter choices and the instationary viscous Burgers equation, i.e. quadratically nonlinear transport equations.

The a posteriori estimator between the RB approximation $u_{N}(\mu)$ and FE approximation $u_{H}(\mu)$ justifies the approach and allows a more efficient basis construction.

Numerical experiments on a parameter-dependent transport problem, discretized with backward Euler, Newton Method and FEM, demonstrate the applicability of the model reduction technique. We compare the results of the RBM with FEM. The CPU times demonstrate the efficiency, in particular we gain a factor $\frac{1}{66}$ for the CPU time.

References:

[1] N. Jung, Anwendung der Reduzierten Basis Methode auf quadratisch instationäre Transportgleichungen - die instationäre viskose Burgers Gleichung, University of Freiburg, Diplomarbeit, University of Freiburg, May 2008

[2] K. Veroy, and A.T. Patera, Certified real-time solution of the parametrized steady incompressible Navier-Stokes equations: rigorous reduced-basis a posteriori error bounds., Int. J. Numer. Methods Fluids 47 (2005), 8-9, pp. 773-788.

\footnotetext{
${ }^{1}$ Freiburg University, Department of Applied Mathematics, Hermann-Herder-Str. 10, D-79104 Freiburg, Germany,

jung.nadine@mathematik.uni-freiburg.de

${ }^{2}$ Westfälische Wilhelms-Universität Münster, Institut für Numerische und Angewandte Mathematik , Einsteinstr. 62, R. 106 , D-48149 Muenster, Germany,

bhaas_01@uni-muenster.de

3 Abteilung für Angewandte Mathematik, Universität Freiburg, Hermann-Herder-Str.10, 79104

Freiburg,

dietmar@mathematik.uni-freiburg.de
} 